\title{
Revisiting soliton contributions to perturbative amplitudes
}

\author{
Constantinos Papageorgakis ${ }^{a, b}$ and Andrew B. Royston ${ }^{c}$ \\ ${ }^{a}$ NHETC and Department of Physics and Astronomy, \\ Rutgers University, Piscataway, NJ 08854-8019, U.S.A. \\ ${ }^{b}$ CRST and School of Physics and Astronomy, \\ Queen Mary, University of London, E1 4NS, U.K. \\ ${ }^{c}$ George P. \& Cynthia Woods Mitchell Institute for Fundamental Physics and Astronomy, \\ Texas A\&M University, College Station, TX 77843, U.S.A. \\ E-mail: c.papageorgakis@qmul.ac.uk, aroyston@physics.tamu.edu
}

ABSTRACT: It is often said that soliton contributions to perturbative processes in QFT are exponentially suppressed by a form factor. We provide a derivation of this form factor by studying the soliton-antisoliton pair production amplitude for a class of scalar theories with generic soliton moduli. This reduces to the calculation of a matrix element in the quantum mechanics on the soliton moduli space. We investigate the conditions under which the latter leads to suppression. Extending this framework to instanton-solitons in five-dimensional Yang-Mills theory leaves open the possibility that such contributions will not be suppressed.

Keywords: Scattering Amplitudes, Solitons Monopoles and Instantons, Nonperturbative Effects

ARXIV EPRINT: 1404.0016 


\section{Contents}

1 Introduction and summary 1

2 Soliton pair production as a form factor 3

3 Evaluation of the nonrelativistic form factor 5

4 Evaluation of the relativistic form factor $\quad 8$

5 Instanton-solitons in 5D MSYM 14

$\begin{array}{ll}\text { A One-point function and the source-free e.o.m. } & 16\end{array}$

B The $\chi, \pi$ saddle point in the presence of centered moduli 18

\section{Introduction and summary}

According to standard QFT lore, soliton production is exponentially suppressed at small coupling and hence unimportant for perturbative physics. For a theory with a dimensionless effective coupling $g_{\text {eff }}$ such intuition usually comes from the study of the large-order behavior of perturbation theory. The basic premise is that the perturbative expansion is an asymptotic series, which approximates the full answer for a scattering amplitude up to a remainder term,

$$
\mathcal{A}\left(g_{\text {eff }}\right)=\sum_{n=0}^{N-1} A_{n} g_{\text {eff }}^{n}+\mathcal{R}_{N}\left(g_{\text {eff }}\right) .
$$

The approximation is good when $\mathcal{R}_{N} \sim e^{-1 / g_{\text {eff }}}$ and this occurs at large orders $N \sim$ $O\left(1 / g_{\text {eff }}\right), e . g$. see [1]. As such, one would conclude that at small coupling all contributions which cannot be accounted for by perturbation theory are exponentially small.

These arguments are most commonly discussed in the context of instanton contributions to partition functions and correlators. However, they are also applicable to the process of virtual soliton-antisoliton pair creation in the following sense $[2,3]$. If one views the soliton-antisoliton pair as being composed of a large number, $n \sim O\left(1 / g_{\text {eff }}\right)$, of perturbative particles clustered together at distances of order one relative to the inverse energy of the incoming particle, then the contribution of this pair to a perturbative process would be captured by the remainder function in (1.1) rather than the sum.

There is also a second, different picture for the origin of soliton suppression, ${ }^{1}$ which becomes apparent after relating contributions from virtual soliton pairs to the creation of on-shell soliton-antisoliton asymptotic states via the optical theorem. One can construct

\footnotetext{
${ }^{1}$ See [4] for a discussion along these lines.
} 
a natural parameter, in addition to the coupling, from the ratio of the soliton Compton wavelength over its size, $R_{C} / R_{S}$. When $R_{S} \gg R_{C}$ quantum effects are small and the soliton can be treated semiclassically. Since this is the regime of perturbative calculations, it is reasonable to expect that soliton contributions to a given amplitude are suppressed by factors of $e^{-R_{S} / R_{C}} .^{2}$

In the main part of this letter we revisit the above discussion by performing a firstprinciples investigation of soliton contributions in scalar theories, which support solitons with generic moduli spaces. Our primary assumption will be that crossing symmetry applies to processes involving asymptotic soliton states and moreover that it continues to hold in the semiclassical approximation. This is e.g. true for the kink solution in the sineGordon model [5]. We begin by employing the optical theorem to associate a soliton loop in a perturbative process with the soliton-antisoliton pair production amplitude. Crossing symmetry then maps the calculation to a form factor in the soliton background, which to leading order reduces to a matrix element in the quantum mechanics on soliton moduli space. This in turn leads to the derivation of a faster-than-any-power suppression factor in $R_{C} / 2 R_{S}$ for such amplitudes sufficiently above threshold. ${ }^{3}$

In line with the soliton literature, we assume that the Compton wavelength, i.e. inverse mass, scales as $g^{2}$ relative to a fixed length scale determined from the potential, such as the inverse mass of a perturbative excitation: $R_{C} \propto g^{2} m^{-1}$. Thus, if the soliton size is also fixed in terms of this mass scale such that $R_{S} \propto m^{-1}$, then faster-than-any-power suppression in $R_{C} / 2 R_{S}$ means suppression relative to perturbative effects. For shorthand we will express functions of this type through the typical example $e^{-2 R_{S} / R_{C}}$, although there are of course other functions with this property. We stress that all such functions lead to contributions that are suppressed compared to any finite order in perturbation theory, provided the size $R_{S}$ is tied to a fixed scale.

However, our formalism also allows for solitons with moduli-dependent sizes. Thus the $e^{-2 R_{S} / R_{C}}$ factor, taken at face value, implies finite contributions from configurations with $R_{S} \propto R_{C}$. Of course, when $R_{S} \propto R_{C}$ one expects that the semiclassical approximation breaks down. A more conservative stance would therefore be that small solitons simply invalidate the above arguments for faster-than-any-power suppression. This still leaves open the possibility of a drastic modification to the perturbative expansion and more powerful tools are needed to determine the role of soliton contributions.

We close by sketching an application of this effect to (dyonic) instanton-solitons in 5D Yang-Mills-Higgs theory. We show how the extra assumption of finiteness, motivated for the maximally supersymmetric theory (MSYM) by its conjectural equivalence to the $(2,0)$ superconformal tensor theory in 6D [6, 7], provides a self-consistent (and nontrivial) mechanism in which recently obtained perturbative divergences [8] could be canceled by soliton contributions.

\footnotetext{
${ }^{2}$ In general, $R_{C} / R_{S}$ and $g_{\text {eff }}$ are different parameters. However, note that for the 't Hooft-Polyakov monopole in Yang-Mills-Higgs theory $R_{C}:=1 / M \propto g_{\mathrm{YM}}^{2} / M_{W}$, while $R_{S} \propto 1 / M_{W}$, where $M_{W}$ is the mass of the perturbative $W$-boson. Hence $R_{C} / R_{S} \propto g_{\mathrm{eff}}=g_{\mathrm{YM}}^{2}$. Similar relations hold for the kink in two-dimensional $\Phi^{4}$ theory.

${ }^{3}$ There might be additional effects at threshold affecting the behavior of the form factor, which we have not taken into account.
} 


\section{Soliton pair production as a form factor}

Consider the following class of real scalar field theories in Minkowski space with Lagrangian

$$
L=\frac{1}{g^{2}} \int \mathrm{d} \mathbf{x}\left\{\frac{1}{2} \dot{\Phi} \cdot \dot{\Phi}-\frac{1}{2} \partial_{\mathbf{x}} \Phi \cdot \partial_{\mathbf{x}} \Phi-V(\Phi)\right\} .
$$

We denote by $\mathbf{x}$ a $(D-1)$-dimensional position vector, while $\mathrm{d} \mathbf{x}$ is shorthand for $\mathrm{d}^{D-1} x$. We take the fields to be $\mathbb{R}^{n}$-valued, and · denotes the Euclidean dot product. ${ }^{4}$ Here we assume that the potential has a dimensionless parameter $g$ controlling the perturbative expansion. Then, in terms of canonically normalized fields $\tilde{\Phi}=g^{-1} \Phi$, we have $\tilde{V}(\tilde{\Phi} ; g)=g^{-2} \tilde{V}(g \tilde{\Phi} ; 1)$, while we have also set $V(\Phi)=\tilde{V}(g \tilde{\Phi} ; 1)[9]$.

We are interested in soliton solutions, classically described by localized, finite-energy field configurations and denoted by $\phi$. Although Derrick's theorem $[10,11]$ precludes the existence of soliton solutions in linear sigma models for $D>2$, we will for the time being leave $D$ arbitrary. Doing so will facilitate the extension to theories with gauge interactions where one can have $D>2$. It will be clearly indicated in the text when it becomes necessary to restrict to two dimensions.

For a fixed topological charge sector, ${ }^{5}$ such classical solutions usually come in a smooth family parameterized by a collection of moduli $U^{M}$, where $M=i, m$. In a translationinvariant theory, a subset of these moduli always consist of the center-of-mass position, $\left(U^{i}\right)=\mathbf{X}$. $U^{m}$ then parameterize all remaining 'centered' moduli. We denote the moduli space of solutions for a given, fixed topological charge as $\mathcal{M}$; it represents a local minimum of the energy functional. An example of a simple model in two dimensions with a nontrivial centered moduli space was studied by Rajaraman and Weinberg [12]. Our class of twodimensional models also contains solitons with moduli-dependent sizes; see e.g. $[13,14]$.

In the presence of a soliton a new sector of the quantum theory opens up. This is orthogonal to the vacuum sector since solitons carry a conserved topological charge [9]. Nevertheless, the soliton-sector single-particle states form a subspace of the total singleparticle Hilbert space and one can study processes involving both perturbative particles and solitons as asymptotic states. Soliton states can be chosen to be energy-momentum eigenstates, $|\mathbf{P}\rangle .{ }^{6}$ Note that, in addition to the soliton's momentum, such states can carry extra labels corresponding to eigenvalues of ancillary operators that commute with the Hamiltonian. These depend on the particulars of the theory and will be left implicit for the rest of our discussion.

Let us now study the self-energy of a perturbative particle, or 'meson', of momentum $k$ in the theory (2.1). Through the optical theorem, unitarity of the S-matrix implies that the imaginary part of any amplitude arises from a sum over a complete set of intermediate states, viz.

$$
2 \operatorname{Im} \mathcal{A}(k \rightarrow k)=\sum_{f} \int \mathrm{d} \Pi_{f}|\mathcal{A}(k \rightarrow f)|^{2} .
$$

\footnotetext{
${ }^{4}$ We use italic letters for $D$-dimensional vectors and boldface letters for spatial $(D-1)$-dimensional ones.

${ }^{5}$ The sectors are labeled by homotopy equivalence classes of maps of the $D-2$-sphere at infinity into the vacuum manifold, $M_{\mathrm{vac}}:=\{\Phi \mid V(\Phi)=0\}$.

${ }^{6}$ We capitalize the momenta of solitons in order to distinguish them from perturbative particle momenta.
} 
In the above, $|\mathbf{f}\rangle$ is a generic multi-particle state of the theory, the sum is to be taken over the full Fock space and $\Pi_{f}$ is the measure for the multi-particle phase space. In general, multi-particle states may be constructed from both perturbative and/or solitonic singleparticle states. However, conservation of topological charge dictates that only states $|\mathbf{f}\rangle$ with zero total topological charge will have nonzero overlap with $|\mathbf{k}\rangle$.

It will be enough for our purposes to concentrate on the simplest such intermediate configuration consisting of a single soliton-antisoliton pair of momentum $-P_{i}$ and $P_{f}$ respectively and denoted as $|\mathbf{f}\rangle=\left|\mathbf{P}_{f},-\mathbf{P}_{i}\right\rangle$. We will therefore focus on the soliton pairproduction amplitude:

$$
\mathcal{A}\left(k \rightarrow P_{f},-\bar{P}_{i}\right)
$$

It is unclear how one should proceed with a direct evaluation of such an expression, since there exists no known associated analytic classical solution and hence no semiclassical expansion scheme. ${ }^{7}$ For that reason, we will employ crossing symmetry - one of the main axioms in the analytic S-matrix approach to quantum field theory [17] — to relate the full amplitude for pair production to that of a process where the soliton absorbs the meson

$$
\mathcal{A}\left(k \rightarrow P_{f},-\bar{P}_{i}\right)=\mathcal{A}\left(P_{i}, k \rightarrow P_{f}\right) .
$$

The advantage of this rewriting is that we can now employ semiclassical tools to evaluate the expression on the r.h.s. . Note that (2.4) is an equality between amplitudes in distinct topological sectors.

The amplitudes (2.4) are nontrivial only when the perturbative particle is off-shell. Therefore, the r.h.s. is related to the form factor

$$
i(2 \pi)^{D} \delta^{(D)}\left(k+P_{i}-P_{f}\right) \mathcal{A}\left(P_{i}, k \rightarrow P_{f}\right)=\int \mathrm{d}^{D} x e^{-i k \cdot x}\left\langle\mathbf{P}_{f}\left|\mathrm{~T}\left\{\Phi(x) e^{-i \int \mathrm{d} t^{\prime} H_{I}\left(t^{\prime}\right)}\right\}\right| \mathbf{P}_{i}\right\rangle,
$$

where $H_{I}$ denotes the interaction Hamiltonian. The Hamiltonian obtained from (2.1) is trivially

$$
H=\int \mathrm{d} \mathbf{x}\left\{\frac{g^{2}}{2} \Pi \cdot \Pi+\frac{1}{g^{2}}\left(\frac{1}{2} \partial_{\mathbf{x}} \Phi \cdot \partial_{\mathbf{x}} \Phi+V(\Phi)\right)\right\},
$$

and its semiclassical expansion in the soliton sector was obtained in [18]. The original conjugate pair $(\Phi, \Pi)$ can be related to the new pairs $\left(U^{M}, P_{N}\right),(\chi, \pi)$ through the canonical transformation

$$
\begin{aligned}
& \Phi(x)=\phi(\mathbf{x} ; U)+g \chi(x ; U) \\
& \Pi(x)=\frac{1}{2}\left(a^{M} \partial_{M} \phi(\mathbf{x} ; U)+\partial_{M} \phi(\mathbf{x} ; U) \bar{a}^{M}\right)+\frac{1}{g} \pi(x ; U),
\end{aligned}
$$

and subject to the constraints

$$
F_{1, M}:=\int \mathrm{d} \mathbf{x} \chi \cdot \partial_{M} \phi=0, \quad F_{2, M}:=\int \mathrm{d} \mathbf{x} \pi \cdot \partial_{M} \phi=0,
$$

\footnotetext{
${ }^{7}$ See however $[15,16]$ for an alternative approach to this problem, which would be interesting to compare with the point of view taken here.
} 
which ensure that the fluctuations $\chi, \pi$ are orthogonal to the zero modes $\partial_{M} \phi$. Here we have inserted factors of $g$ so that the fluctuation fields are canonically normalized. The functionals $a^{M}, \bar{a}^{M}$ are given by

$$
a^{N}=\frac{1}{g^{2}}\left(P_{M}-\int \pi \cdot \partial_{M \chi}\right) C^{M N}, \quad \bar{a}^{M}=\frac{1}{g^{2}} C^{M N}\left(P_{M}-\int \partial_{M} \chi \cdot \pi\right),
$$

where $C=(G-g \Xi)^{-1}$ with

$$
\Xi_{M N}=\frac{1}{g^{2}} \int \chi \cdot \partial_{M} \partial_{N} \phi, \quad G_{M N}=\frac{1}{g^{2}} \int \partial_{M} \phi \cdot \partial_{N} \phi
$$

$G_{M N}$ is the metric on moduli space, induced from the flat metric on field configuration space.

In terms of these new variables the Hamiltonian can be written as

$$
H=\frac{g^{4}}{2} a^{M} G_{M N} a^{N}+\mathrm{v}\left(U^{m}\right)+\int\left[\frac{1}{2} \pi \cdot \pi+g s \cdot \chi+\frac{1}{2} \chi \cdot \Delta \chi+V_{I}(\chi)\right]+O\left(g^{2}\right),
$$

with $V_{I}(\chi)$ denoting cubic and higher-order interaction terms in the fluctuations $\chi$ coming from the original potential. In writing the above, we have ignored operator-ordering ambiguities, such that $a^{M}=\bar{a}^{M}+O\left(g^{2}\right)$. These corrections correspond to two-loop effects that will not be important for the rest of our calculation.

We have also defined

$$
\begin{aligned}
& s\left(\mathbf{x} ; U^{m}\right):=\frac{1}{g^{2}}\left(-\partial_{\mathbf{x}}^{2} \phi+\left.\frac{\partial V}{\partial \Phi}\right|_{\Phi=\phi}\right), \quad \Delta:=-\delta_{a b} \partial_{\mathbf{x}}^{2}+\left.\frac{\delta^{2} V}{\delta \Phi \delta \Phi}\right|_{\Phi=\phi}, \\
& \mathrm{v}\left(U^{m}\right):=\frac{1}{g^{2}} \int \mathrm{d} \mathbf{x}\left(\frac{1}{2} \partial_{\mathbf{x}} \phi \cdot \partial_{\mathbf{x}} \phi+V(\phi)\right)=M_{\mathrm{cl}}+\delta \mathrm{v}\left(U^{m}\right) .
\end{aligned}
$$

If $\phi$ is an exact solution to the time-independent equations of motion then $s\left(\mathbf{x} ; U^{m}\right)=0$ and $\delta \mathrm{v}\left(U^{m}\right)=0$. However in theories with centered moduli it is sometimes convenient to expand around a configuration that is only an approximate solution. This will induce a tadpole for $\chi$ and a moduli-dependent potential.

\section{Evaluation of the nonrelativistic form factor}

We will now use standard techniques to evaluate the form factor (2.5) in the regime of small soliton velocities. The semiclassical expansion of the Hamiltonian around a slowly-moving soliton configuration follows from (2.11):

$$
H=H^{(-2)}+H^{(0)}+O(g),
$$

where

$$
\begin{aligned}
H^{(-2)} & =M_{\mathrm{cl}}, \\
H^{(0)} & =\frac{1}{2} P_{M} G^{M N} P_{N}+\delta \mathrm{v}(U)+\frac{1}{2} \int(\pi \cdot \pi+\chi \cdot \Delta \chi),
\end{aligned}
$$


This expansion is valid provided we are in the small-velocity and small-(moduli space) potential approximation:

$$
P_{M} \sim O(1 / g), \quad s\left(\mathbf{x} ; U^{m}\right) \sim O(1) \quad \Rightarrow \quad M_{\mathrm{cl}} \sim O\left(1 / g^{2}\right) \text { and } \delta \mathrm{v}\left(U^{m}\right) \sim O(1) .
$$

These conditions ensure that $\phi\left(\mathbf{x} ; U^{M}(t)\right)$ is an approximate classical solution to the timedependent equations of motion, in such a way that the corrections incurred from not expanding around an exact solution are comparable to the corrections incurred from the semiclassical saddle-point approximation itself; see [18] for further details. Given (3.3) and the fact that $G^{M N} \sim O\left(g^{2}\right)$, one can check that the $H^{(n)}$ terms in (3.1) are $O\left(g^{n}\right)$.

It is now easy to see that the leading contributions to the form factor are

$$
\begin{aligned}
\int \mathrm{d}^{D} x e^{-i k \cdot x} & \left\langle\mathbf{P}_{f}\left|\mathrm{~T}\left\{\Phi(x) e^{-i \int_{-T}^{T} \mathrm{~d} t^{\prime} H_{I}\left(t^{\prime}\right)}\right\}\right| \mathbf{P}_{i}\right\rangle \\
& =\int \mathrm{d}^{D} x e^{-i k \cdot x}\left\langle\mathbf{P}_{f}, T|\phi(\mathbf{x} ; U(t))| \mathbf{P}_{i},-T\right\rangle+O(g) \\
& =\int \mathrm{d}^{D} x e^{-i k \cdot x}\left\langle\mathbf{P}_{f}\left|e^{i H(t-T)} \phi(\mathbf{x} ; U) e^{-i H(t+T)}\right| \mathbf{P}_{i}\right\rangle+O(g) .
\end{aligned}
$$

The latter admits further simplification to leading order where the dynamics reduce to quantum mechanics on the $d$-dimensional soliton moduli space $\mathcal{M}$ [18]. This can be straightforwardly seen by noting that the last two terms in $H^{(0)}$ simply renormalize $M_{\mathrm{cl}}+$ $\delta \mathrm{v}(U)$ to yield

$$
\hat{H}_{\text {s.c. }}=M_{1-\text { loop }}+\frac{1}{2} \hat{P}_{M} \hat{G}^{M N} \hat{P}_{N}+\delta \hat{\mathrm{v}}_{1-\text { loop }} .
$$

In the above we have used $\hat{G}^{M N}=G^{M N}(\hat{U})$ and placed hats on $\left(U^{M}, P_{N}\right)$ to emphasize the fact that they are operators, satisfying standard commutation relations $\left[\hat{U}^{M}, \hat{P}_{N}\right]=i \delta^{M}{ }_{N}$.

We take a canonical approach to this quantum mechanics where stationary states are represented by wavefunctions $\Psi(U)$ on moduli space, such that $\left(\hat{U}^{M} \Psi\right)(U)=U^{M} \Psi(U)$ and $\left(\hat{P}_{M} \Psi\right)(U)=-i \partial_{M} \Psi(U)$. In particular the state $|\mathbf{P}\rangle$ may be expanded as

$$
|\mathbf{P}\rangle=\int \mathrm{d}^{d} U \sqrt{G} \Psi_{\mathbf{P}}(U)|U\rangle .
$$

Note that $\mathbf{P}$ is the set of eigenvalues of the center-of-mass momentum operators, $\hat{P}_{i}$. Translational invariance implies that the full moduli space takes a factorized form,

$$
\mathcal{M}=\mathbb{R}_{\mathbf{X}}^{D-1} \times \tilde{\mathcal{M}}
$$

with metric

$$
\mathrm{d} s^{2}=M_{\mathrm{cl}} \mathrm{d} \mathbf{X} \cdot \mathrm{d} \mathbf{X}+\tilde{G}_{m n} \mathrm{~d} U^{m} \mathrm{~d} U^{n} .
$$

The first factor is parameterized by the center-of-mass moduli $\left(U^{i}\right)=\mathbf{X}$, while the centered moduli space $\tilde{\mathcal{M}}$ is parameterized by the remaining moduli $U^{m}$. Additionally, translational invariance implies that the moduli space potential $\delta \mathrm{v}$ is independent of the $U^{i}$. It then follows that the $\hat{P}_{i}$ commute with the Hamiltonian (3.5) and we can choose our wavefunctions to be simultaneous eigenfunctions of energy and center-of-mass momentum. We will 
denote the energy eigenvalues as $E_{\mathbf{P}}$, so that $e^{i \hat{H}_{\text {s.c. }} t} \Psi_{\mathbf{P}}=e^{i E_{\mathbf{P}} t} \Psi_{\mathbf{P}}$. Given (3.5) and (3.8), the energy eigenvalues take the form

$$
E_{\mathbf{P}}=M_{1-\text { loop }}+\frac{1}{2 M_{\mathrm{cl}}} \mathbf{P}^{2}+\tilde{E}
$$

where $\tilde{E}$ represents the contribution to the energy of the state from the dynamics on the centered moduli space. Note that the kinetic energy term contributes at the same order, $O(1)$, as the one-loop correction to the rest mass, per (3.3). As mentioned at the beginning of our analysis, there might be additional labels characterizing the soliton state corresponding to extra operators that commute with the Hamiltonian, in which case they also characterize the wavefunction.

We can now explicitly write the matrix element appearing in (3.4) as

$$
\begin{aligned}
\left\langle\mathbf{P}_{f}\right| e^{i H(t-T)} \phi & (\mathbf{x} ; U) e^{-i H(t+T)}\left|\mathbf{P}_{i}\right\rangle \\
& =e^{-i\left(E_{i}+E_{f}\right) T} e^{-i\left(E_{i}-E_{f}\right) t} \int \mathrm{d}^{d} U \sqrt{G} \Psi_{f}^{*} \phi(\mathbf{x} ; U) \Psi_{i}+O(g) .
\end{aligned}
$$

The factorization of the moduli space (3.8) together with the $\mathbf{X}$-independence of the potential $\delta \mathrm{v}(U)$, imply that the wavefunctions also factorize accordingly:

$$
\Psi_{\mathbf{P}}\left(U^{M}\right)=\frac{1}{M_{\mathrm{cl}}^{(D-1) / 2}} e^{i \mathbf{P} \cdot \mathbf{X}} \tilde{\Psi}_{\mathbf{P}}\left(U^{m}\right) .
$$

The $\tilde{\Psi}$ are wavefunctions on the centered moduli space. In general we will denote quantities associated with the centered part of the moduli space with a tilde.

Translational invariance implies that the soliton solution depends on the center-of-mass moduli only through the difference $\mathbf{x}-\mathbf{X}$, so that $\phi\left(\mathbf{x} ; U^{M}\right)=\phi\left(\mathbf{x}-\mathbf{X} ; U^{m}\right)$. Using this fact, along with the factorized form of the wavefunctions, we have

$$
\begin{aligned}
& \left\langle\mathbf{P}_{f}\left|e^{i H(t-T)} \phi(\mathbf{x} ; U) e^{-i H(t+T)}\right| \mathbf{P}_{i}\right\rangle \\
& \quad=e^{-i\left(E_{i}+E_{f}\right) T} e^{-i\left(E_{i}-E_{f}\right) t} \int_{\mathrm{d} \mathbf{X} e^{i\left(\mathbf{P}_{i}-\mathbf{P}_{f}\right) \cdot \mathbf{X}} \int_{\tilde{\mathcal{M}}} \mathrm{d} U \sqrt{\tilde{G}} \tilde{\Psi}_{f}^{*} \phi\left(\mathbf{x}-\mathbf{X} ; U^{m}\right) \tilde{\Psi}_{i}+O(g)} \\
& \quad=e^{-i\left(E_{i}+E_{f}\right) T} e^{-i\left(P_{i}-P_{f}\right) \cdot x} \int_{\tilde{\mathcal{M}}} \mathrm{d} U \sqrt{\tilde{G}} \tilde{\Psi}_{f}^{*} \mathcal{F}[\phi] \tilde{\Psi}_{i}+O(g)
\end{aligned}
$$

where by $\mathcal{F}[\phi]=\mathcal{F}[\phi]\left(\mathbf{P}_{f}-\mathbf{P}_{i} ; U^{m}\right)$ we denote the Fourier transform of $\phi$ with respect to its first, spatial argument, and we have introduced the Lorentz spacetime momenta $P_{i, f}=\left(E_{i, f}, \mathbf{P}_{i, f}\right)$.

Inserting (3.12) into (3.4) and carrying out the integral over spacetime produces the energy-momentum conserving delta function, $(2 \pi)^{D} \delta^{(D)}\left(k+P_{i}-P_{f}\right)$. Hence, from (2.5), the leading semiclassical expression for the amplitude of interest is given by a quantum mechanical matrix element of the Fourier transform $\mathcal{F}[\phi]$ on the centered moduli space

$$
\mathcal{A}\left(P_{i}, k \rightarrow P_{f}\right)=-i e^{-i\left(E_{i}+E_{f}\right) T} \int_{\tilde{\mathcal{M}}} \mathrm{d} U \sqrt{\tilde{G}} \tilde{\Psi}_{f}^{*} \mathcal{F}[\phi]\left(\mathbf{k} ; U^{m}\right) \tilde{\Psi}_{i}+O(g) .
$$


In the special case where there are no centered moduli, (3.13) reduces to the known result that the form factor is just $\mathcal{F}[\phi]$, up to an energy-momentum-preserving $\delta$-function [9] with standard initial and final state normalization factors.

Let us now comment on the validity of our calculation. Note that the result (3.13) was obtained in the small-velocity approximation (3.3); the form of the energy eigenvalues (3.9) provides a clear manifestation of the nonrelativistic limit. However, the regime needed to extract information about the pair-creation process through crossing symmetry requires large velocity exchange and hence momentum transfer of the order of the soliton mass. One may be tempted to express the answer in terms of the Lorentz-invariant quantity

$$
k^{2}=\left(P_{f}-P_{i}\right)^{\mu}\left(P_{f}-P_{i}\right)_{\mu},
$$

which in the small velocity approximation leads to

$$
\sqrt{-k^{2}}=|\mathbf{k}|\left(1+O\left(\mathbf{P}_{i}^{2} / M_{\mathrm{cl}}^{2}, \mathbf{P}_{f}^{2} / M_{\mathrm{cl}}^{2}\right)\right)
$$

and assume that making the replacement $|\mathbf{k}| \rightarrow \sqrt{-k^{2}}$ correctly captures all relativistic corrections to (3.13). If this were true, then one could extend the result (3.13) to large spacelike $k^{2}$, corresponding to large values of the argument of the Fourier transform. Assuming smoothness of the classical soliton profile, the Riemann-Lebesgue lemma would imply that the Fourier transform falls off faster than any power. Then one could consider the analytic continuation from spacelike to timelike $k^{2}$ and attempt to draw a conclusion about the pair production process, as in [4]. However, as we will see, this procedure does not properly account for the large-velocity corrections. An indication of this is that it leads to form factors that fall off faster than any power in momentum transfer. This is a result that is qualitatively incompatible with expectations from any asymptotically free theory, where the large momentum behavior of amplitudes is expected to be power-law. See e.g. [19] for a discussion in the context of skyrmion form factors in QCD.

In the case of the two-dimensional kink in $\Phi^{4}$ theory, seminal work by Gervais, Jevicki and Sakita [20] showed how velocity corrections can be systematically accounted for to recover the covariant expression for the soliton energy, $M_{\mathrm{cl}} \rightarrow \sqrt{\mathbf{P}^{2}+M_{\mathrm{cl}}^{2}}$. This answer is to be expected, since the starting point is a Lorentz-invariant theory. In the next section we will show how the same techniques can be applied in the more general class of Lorentzinvariant theories considered here. We will be interested in evaluating the form factor (2.5) rather than the soliton energy. Fortunately, the techniques of [20] have been adapted to this context by [21], the methodology of which we will be following closely.

\section{Evaluation of the relativistic form factor}

We now proceed to evaluate the form factor for processes involving large velocity exchange. The path integral formulation is much more appropriate for the purposes of resumming the relativistic corrections and we will favor it over of the canonical approach implemented thus far. The two qualitative differences between the general case and the kink in $\Phi^{4}$ theory as considered by [20] are, first, a lack of an explicit classical soliton solution to work with 
and, second, the possible presence of centered moduli. Both can be taken into account and their discussion can be appropriately modified, provided we continue to make the simplifying assumptions of the Manton (small-velocity and small moduli-space-potential) approximation for the dynamics of the centered moduli. Specifically, we will impose

$$
\begin{aligned}
& P_{m} / m \sim O(1 / g), \quad s\left(\mathbf{x} ; U^{m}\right) \sim O(1) \quad \text { and } \quad \delta \mathrm{v}\left(U^{m}\right) \sim O(1) \\
& \text { but we take } \quad \mathbf{P} / m \sim O\left(1 / g^{2}\right) \quad \text { such that } \quad \mathbf{P} \propto M_{\mathrm{cl}} .
\end{aligned}
$$

At the end of this section we will comment on the possibility of extending our results when these assumptions are relaxed.

The transition amplitude from an initial state $i$ described by $\Psi_{i}\left(U^{M}(-T) ; \chi\right)$ to a final state $f$ described by $\Psi_{f}\left(U^{M}(T) ; \chi\right)$ is

$$
\begin{aligned}
S_{f i} & =\int[D U D P D \chi D \pi] \delta\left(F_{1}\right) \delta\left(F_{2}\right) e^{i \int_{-T}^{T} \mathrm{~d} t^{\prime} L} \Psi_{f}^{*} \Psi_{i}, \quad \text { with } \\
L & =P_{M} \dot{U}^{M}+\int \mathrm{d} \mathbf{x} \pi \dot{\chi}-H .
\end{aligned}
$$

An incoming soliton state of momentum $\mathbf{P}_{i}$ is defined by taking $\Psi_{i}=e^{i \mathbf{P}_{i} \cdot \mathbf{X}_{i}} \tilde{\Psi}_{i}\left(U^{m}\right)$, where $\mathbf{X}_{i}=\mathbf{X}(-T)$, and similarly for outgoing soliton states. We remind that the $\tilde{\Psi}_{i, f}$ are wavefunctions on the centered moduli space. We can consider time-ordered correlators of the meson field between soliton states by inserting appropriate factors of $\Phi\left(x_{1}\right) \cdots \Phi\left(x_{n}\right)$ under the path integral, and using the relation $\Phi(x)=\phi\left(\mathbf{x}-\mathbf{X}(t) ; U^{m}\right)+g \chi\left(t, \mathbf{x}-\mathbf{X}(t) ; U^{m}\right)$.

We are interested in the particular case of the 1-point function and hence in

$$
\begin{aligned}
\left\langle\mathbf{P}_{f}, T|\Phi(x)| \mathbf{P}_{i},-T\right\rangle=\int[D \mathbf{X} D \mathbf{P}] e^{i\left(\mathbf{X}_{i} \cdot \mathbf{P}_{i}-\mathbf{X}_{f} \cdot \mathbf{P}_{f}\right)} \int\left[D U^{m} D P_{n}\right] \tilde{\Psi}_{f}^{*} \tilde{\Psi}_{i} \times \\
\times \int[D \chi D \pi] \delta\left(F_{1}\right) \delta\left(F_{2}\right) e^{i \int_{-T}^{T} \mathrm{~d} t^{\prime} L} \Phi[U, P ; \chi](x) .
\end{aligned}
$$

Let us focus first on the internal path integral over $\chi$ and $\pi$ for which we will proceed to compute the leading contribution at small $g$. This was done in [20] for the case of the 0 point function by evaluating the action on the saddle-point solution for $\chi, \pi$ corresponding to the moving soliton. We argue in appendix A that the same saddle point solution gives the leading contribution to the one-point function, even though one should now be solving the equations of motion with source. This is a special feature of working with the onepoint function and would not be true for higher point functions. A completely analogous discussion can be found in the papers of Dorey et.al [21, 22]. We denote this saddle point $\left(\chi_{\mathrm{cl}}, \pi_{\mathrm{cl}}\right)$ and expand the fields as $\chi=\chi_{\mathrm{cl}}+\delta \chi, \pi=\pi_{\mathrm{cl}}+\delta \pi$.

Before continuing with the details, we wish to emphasize one property of the semiclassical limit in which we work. One approach to the computation would be the following. One could work around a saddle point of the Hamiltonian (2.11), taking $g$ to be small, while holding $\mathbf{P}$ fixed - the soliton momenta enter (2.11) through (2.9). Thus, one would effectively be sending $g \rightarrow 0$, carrying out the computation for arbitrary $\mathbf{P}_{i, f}$, and then at the end one could consider the limit of the result as the transfer $\Delta \mathbf{P} / m=\left(\mathbf{P}_{f}-\mathbf{P}_{i}\right) / m \rightarrow \infty$. (Here we inserted a factor of the meson mass to get a dimensionless quantity.) However 
this is not the correct limit to consider if one wishes to access the theory in the regime related to pair creation by crossing symmetry. Rather one should be sending $g \rightarrow 0$ and $\Delta \mathbf{P} / m \rightarrow \infty$ simultaneously, while holding the velocity transfer $\Delta v \sim \Delta \mathbf{P} / M \sim g^{2} \Delta \mathbf{P} / m$ fixed and $O(1){ }^{8}$ Thus, it is important in the following that the momentum is treated as an $O\left(g^{-2}\right)$ quantity - i.e. the same order as the soliton mass. This is indeed what we do, and it is also an important, but unstated, assumption in the original analysis of [20].

Now let us return to the computation. Starting with the Hamiltonian (2.11) one can find a saddle-point solution to the $\chi, \pi$ equations of motion perturbatively in $g$ by making use of the scaling assumptions for the momenta (4.1). The details of this calculation are carried out in appendix B. One finds

$$
\chi_{\mathrm{cl}}=g^{-1} \phi\left(\Lambda(\mathbf{x}-\mathbf{X}) ; U^{m}\right)-g^{-1} \phi\left((\mathbf{x}-\mathbf{X}) ; U^{m}\right)+O(1),
$$

where

$$
\Lambda^{i}{ }_{j}=\delta^{i}{ }_{j}+\left(\sqrt{1+\frac{\mathbf{P}^{2}}{M_{\mathrm{cl}}^{2}}}-1\right) \frac{P^{i} P_{j}}{\mathbf{P}^{2}}
$$

is a Lorentz contraction factor. The insertion can then be expressed as

$$
\Phi=\phi\left(R_{S}^{-1} \Lambda(\mathbf{x}-\mathbf{X}) ; U^{m}\right)+O(g) \equiv \Phi_{\mathrm{cl}}+O(g) .
$$

The quantity $R_{S}$, inserted in the argument of the classical soliton solution on dimensional grounds, characterizes the size of the soliton. For example, in $\Phi^{4}$ theory $R_{S}=1 / m$, with $m$ the meson mass. As we previously indicated, in the general class of theories considered here it can in principle be a function of the centered moduli.

With this solution in hand, we want to evaluate (4.3) in the presence of centered moduli. For this, we also need the Lagrangian evaluated on the solution:

$$
L=\mathbf{P} \cdot \dot{\mathbf{X}}-\sqrt{\mathbf{P}^{2}+M_{\mathrm{cl}}^{2}}+L^{(0)}\left[U^{m}, P_{m} ; \delta \chi, \delta \pi ; \mathbf{P}\right]+L_{\mathrm{int}},
$$

where $L_{\text {int }}$ starts at $O(g)$ and

$$
L^{(0)}=P_{m} \dot{U}^{m}-\tilde{H}_{\mathrm{eff}}\left[U^{m}, P_{m} ; \mathbf{P}\right]
$$

is an $O(1)$ contribution describing the dynamics of the centered moduli, whose precise form we will not require. $\tilde{H}_{\text {eff }}$ includes the 1-loop potential from integrating out the fluctuation fields $(\delta \chi, \delta \pi)$. The leading contribution to (4.3) then takes the form

$$
\begin{aligned}
\left\langle\mathbf{P}_{f}|\Phi(x)| \mathbf{P}_{i}\right\rangle=\lim _{T \rightarrow \infty} \int[D & \mathbf{X} D \mathbf{P}] e^{i\left(\mathbf{X}_{i} \cdot \mathbf{P}_{i}-\mathbf{X}_{f} \cdot \mathbf{P}_{f}\right)} e^{i \int_{-T}^{T} \mathrm{~d} t^{\prime}\left(\mathbf{P} \cdot \dot{\mathbf{X}}-\sqrt{\mathbf{P}^{2}+M_{\mathrm{cl}}^{2}}\right)} \times \\
& \times \int_{\tilde{\mathcal{M}}} \mathrm{d} U \sqrt{\tilde{G}} \tilde{\Psi}_{f}^{*}\left(U^{m} ; \mathbf{P}(T)\right) \times \\
& \times \Phi_{\mathrm{cl}}\left[\mathbf{x}-\mathbf{X}(t), \mathbf{P}(t) ; U^{m}\right] \tilde{\Psi}_{i}\left(U^{m} ; \mathbf{P}(-T)\right)(1+O(g)) .
\end{aligned}
$$

\footnotetext{
${ }^{8}$ We thank E. Witten for communication on this point.
} 
In the above we have expressed the centered moduli space path integral as a position-basis matrix element in the quantum mechanics on the centered moduli space with Hamiltonian $\tilde{H}_{\text {eff. Note that the }}(\mathbf{X}, \mathbf{P})$ path integral is a functional integral representation of the quantum mechanics for a relativistic particle. From the point of view of the translational moduli space dynamics, $U^{m}$ are merely parameters, so we can carry out the functional integration over $\mathbf{X}$ and $\mathbf{P}$ first and then integrate over the centered moduli space.

Thus the quantity that we would like to study is

$$
\begin{aligned}
& \lim _{T \rightarrow \infty} \int[D \mathbf{X} D \mathbf{P}] e^{i\left(\mathbf{X}_{i} \cdot \mathbf{P}_{i}-\mathbf{X}_{f} \cdot \mathbf{P}_{f}\right)} e^{i \int_{-T}^{T} \mathrm{~d} t^{\prime}\left(\mathbf{P} \cdot \dot{\mathbf{X}}-\sqrt{\mathbf{P}^{2}+M_{\mathrm{cl}}^{2}}\right)} \mathcal{O}, \\
& \quad \text { where } \quad \mathcal{O}=\tilde{\Psi}_{f}^{*}\left(U^{m} ; \mathbf{P}(T)\right) \Phi_{\mathrm{cl}}\left[\mathbf{x}-\mathbf{X}(t), \mathbf{P}(t) ; U^{m}\right] \tilde{\Psi}_{i}\left(U^{m} ; \mathbf{P}(-T)\right) .
\end{aligned}
$$

This is a phase-space path integral for a relativistic particle (soliton) of mass $M_{\mathrm{cl}}$ with the insertion $\mathcal{O}$ involving the boosted classical soliton profile (4.6) with (4.5). In this expression the gauge symmetry of worldline reparameterizations has been fixed by identifying the coordinate time $t^{\prime}$ with the embedding coordinate $X^{0}-i$.e. static gauge. As a result Lorentz invariance is obscured. In fact, this gauge-fixing condition is not admissible as pointed out in [23] and, indeed, if one proceeds with the naive evaluation of (4.6) one finds a result that is not Lorentz invariant.

The proper way to evaluate the transition function in a manifestly covariant manner has been well studied [24-26]. In a forthcoming work [27] we discuss how to extend this calculation to the case of the one-point function (4.10). The crucial observation is that in two dimensions the argument of the classical soliton profile is a gauge-fixed form of the gauge-invariant quantity

$$
\begin{aligned}
\frac{1}{M_{\mathrm{cl}}} \epsilon_{\mu \nu} P^{\mu}(x-X)^{\nu}= & \frac{1}{M_{\mathrm{cl}}}\left(P^{0}(\mathbf{x}-\mathbf{X})-\mathbf{P}\left(t-X^{0}\right)\right) \\
\stackrel{\text { static gauge }}{\longrightarrow} & \frac{1}{M_{\mathrm{cl}}} \sqrt{\mathbf{P}^{2}+M_{\mathrm{cl}}^{2}}(\mathbf{x}-\mathbf{X})=\Lambda(\mathbf{x}-\mathbf{X}) .
\end{aligned}
$$

Using the above and employing the techniques of [21, 27], we find that (4.9) takes the following form to leading order:

$$
\left\langle\mathbf{P}_{f}|\Phi(x)| \mathbf{P}_{i}\right\rangle \propto e^{-i\left(P_{f}-P_{i}\right) \cdot x} \int_{\tilde{\mathcal{M}}} \mathrm{d} U \sqrt{\tilde{G}} \tilde{\Psi}_{f}^{*} \mathcal{F}[\phi]\left(\frac{2 R_{S}\left(U^{m}\right)}{R_{C}} \zeta\left(P_{f}, P_{i}\right)\right) \tilde{\Psi}_{i},
$$

where $\mathcal{F}[\phi](u)=\int \mathrm{d} v e^{-i u v} \phi(v)$ is the Fourier transform of the classical soliton profile, $\tilde{\Psi}_{i, f}=\tilde{\Psi}_{i, f}\left(U^{m} ; P_{i, f}\right)$ and

$$
\zeta\left(P_{f}, P_{i}\right):=\frac{2 \epsilon_{\mu \nu} P_{f}^{\mu} P_{i}^{\nu}}{\left(P_{f}+P_{i}\right)^{2}} .
$$

In (4.12) $R_{C}=1 / M_{\mathrm{cl}}$ is the soliton Compton wavelength and we have neglected multiplicative prefactors - this simplification will be justified momentarily. One can then easily relate this matrix element to the amplitude (2.5):

$$
\mathcal{A}\left(P_{i}, k \rightarrow P_{f}\right) \propto \int_{\tilde{\mathcal{M}}} \mathrm{d} U \sqrt{\tilde{G}} \tilde{\Psi}_{f}^{*} \mathcal{F}[\phi]\left(\frac{2 R_{S}\left(U^{m}\right)}{R_{C}} \zeta\left(P_{f}, P_{i}\right)\right) \tilde{\Psi}_{i}
$$


Now, given that the classical soliton profile $\phi$ is a smooth $\left(C^{\infty}\right)$ function of its position argument, we can draw a rather strong conclusion about the asymptotic behavior of the Fourier transform in (4.14). For any values of momenta such that $\zeta$ is not $O\left(g^{2}\right)$ or smaller, it is the $2 R_{S} / R_{C}$ factor that controls the parametric size of the argument of the Fourier transform. Given this, and as long as the soliton size is bounded away from zero, $R_{S}^{\min }>0$, we will have that $\left(2 R_{S} / R_{C}\right)|\zeta| \rightarrow \infty$ in the semiclassical limit. Here we remind that $R_{C}^{-1}=M_{\mathrm{cl}}$ is assumed to be $O\left(1 / g^{2}\right)$ relative to some fixed mass scale determined from the potential (e.g. the meson mass). The Riemann-Lebesgue lemma then implies that ${ }^{9}$

$$
\mathcal{F}[\phi]\left(\frac{2 R_{S}\left(U^{m}\right)}{R_{C}} \zeta\right) \sim e^{-\frac{2 R_{S}\left(U^{m}\right)}{R_{C}}|\zeta|} \text { as } \quad\left(2 R_{S} / R_{C}\right)|\zeta| \rightarrow \infty .
$$

Let us emphasize that the exponential on the r.h.s. is a typical function exhibiting a fasterthan-any-power falloff. It is used for concreteness, but the exact expression will depend on the details of the theory under consideration. In any case, the important property for our purposes is the faster-than-any-power falloff. This asymptotic behavior is unaffected by the prefactors that we neglected starting at (4.12).

This leads to the asymptotic estimate

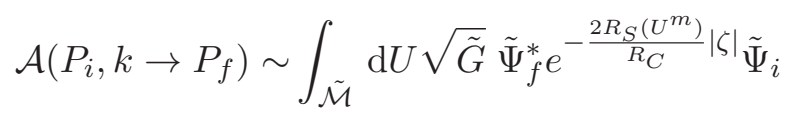

for the leading contribution to the form factor as $g \rightarrow 0$. Note that the centered moduli space represents the internal degrees of freedom of the single-particle state. A field theory interpretation requires a single-particle state to have a finite number of internal degrees of freedom. The eigenvalues labeling them should be discrete eigenvalues of the centeredmoduli-space Hamiltonian $\tilde{H}_{\text {eff }}$. Hence the wavefunctions on the centered moduli space $\tilde{\Psi}$ should be $L^{2}$; this is automatically the case if $\tilde{\mathcal{M}}$ is compact. Then we have the inequalities

$$
\begin{aligned}
\int_{\tilde{\mathcal{M}}} \mathrm{d} U \sqrt{\tilde{G}} \tilde{\Psi}_{f}^{*} e^{-\frac{2 R_{S}\left(U^{m}\right)}{R_{C}}|\zeta|} \tilde{\Psi}_{i} & \leq \int_{\tilde{\mathcal{M}}} \mathrm{d} U \sqrt{\tilde{G}}\left|\tilde{\Psi}_{f}^{*} \tilde{\Psi}_{i}\right| e^{-\frac{2 R_{S}\left(U^{m}\right)}{R_{C}}|\zeta|} \\
& \leq e^{-\frac{2 R_{S}^{\min }}{R_{C}}|\zeta|}|| \tilde{\Psi}_{f}^{*} \tilde{\Psi}_{i} \|_{L^{1}} \\
& \leq e^{-\frac{2 R_{S}^{\min }}{R_{C}}|\zeta|}|| \tilde{\Psi}_{f}\left\|_{L^{2}}|| \tilde{\Psi}_{i}\right\|_{L^{2}} \\
& =e^{-\frac{2 R_{S}^{\min }}{R_{C}}|\zeta|}
\end{aligned}
$$

where in the second-last step we used Hölder's inequality. Hence we have reached the result

$$
\mathcal{A}\left(P_{i}, k \rightarrow P_{f}\right) \lesssim e^{-\frac{2 R_{S}^{\min }}{R_{C}}|\zeta|}
$$

As we first discussed in the nonrelativistic case, in order to use crossing symmetry to obtain the answer for the pair production amplitude we need to first express $\zeta$ in terms

\footnotetext{
${ }^{9}$ As stated by the Riemann-Lebesgue lemma, the Fourier transform $\mathcal{F}[f](p)$ of an $L^{1}$-function $f(x)$ goes to zero as $|p| \rightarrow \infty$. Accordingly, if $f(x)$ is $C^{\infty}, \mathcal{F}\left[f^{(n)}\right](p)=(i p)^{n} \mathcal{F}[f](p)$ should also go to zero as $p \rightarrow \infty$; i.e. $\mathcal{F}[f](p)$ goes to zero faster than any power.
} 
of the momentum transfer $k^{2}=\left(P_{f}-P_{i}\right)^{2}$. Using the fact that $P_{i, f}$ are on shell, one can show that

$$
\zeta=\sqrt{\frac{k^{2}}{k^{2}-4 M_{\mathrm{cl}}^{2}}} .
$$

The above result is consistent with expectations. First, on physical grounds the form factor should be a function of the momentum transfer only; $\zeta=\zeta\left(k^{2}\right)$. Second, as $k^{2} \rightarrow \infty$ we expect $\zeta\left(k^{2}\right) \rightarrow O(1)$; otherwise, one would obtain an amplitude with exponential behavior for large $k^{2}$, in contradiction with the large-momentum behavior of asymptotically free theories. Finally, it agrees exactly with the prescription proposed in [19] in the context of the Skyrme model, where it was also observed that exponential falloff is inconsistent with asymptotic freedom.

This quantity can now be analytically continued from spacelike to timelike $k^{2}$. Thus, via crossing symmetry we finally arrive at

$$
\mathcal{A}\left(k \rightarrow-P_{i}, P_{f}\right) \lesssim e^{-\frac{2 R_{S}^{\min }}{R_{C}} \zeta}
$$

where the physical region of interest is for $k^{2}>4 M_{\mathrm{cl}}^{2}$. This is our main result, in agreement with the original expectation from dimensional analysis. Note that if $R_{S}^{\min }$ is of order $R_{C}$ this does not lead to suppression.

Some comments on this result are in order:

- We would like to emphasize that in deriving (4.20) we have worked in a fixed topological charge sector, labeled by $\gamma \in \pi_{D-2}\left(M_{\mathrm{vac}}\right)$; see footnote 5 . Thus quantities such as $\mathcal{M}$ and $R_{S}$ depend on $\gamma$. In order to conclude that all soliton contributions to the perturbative process (2.2) are exponentially suppressed, one must demonstrate that the r.h.s. of $(2.2)$ is suppressed for all those charges $\gamma$ such that $\tilde{\mathcal{M}}(\gamma)$ supports single-particle soliton states.

- Furthermore, one would like to show that (4.20) holds for all possible choices of additional quantum numbers associated with internal degrees of freedom. We have not shown this for quantum numbers violating the scaling assumptions (4.1). In terms of the energy of the soliton state, those assumptions correspond to considering contributions from translational motion that are $O\left(g^{-2}\right)$, while restricting contributions from 'motion' in the internal directions to be $O(1)$. On the one hand, this can be relaxed for flat internal moduli, which are typically associated with a conserved charge. A saddle point solution analogous to the boosted soliton profile can then be obtained; see e.g. [21]. On the other hand, when the internal moduli space has curvature, radiation effects are important and the methods we have employed here, i.e. splitting the degrees of freedom into collective coordinates and oscillators, are not expected to be useful.

- As we are working with an asymptotic form of the Fourier transform of the soliton profile, one might worry that Stokes phenomena will be important for this analytic 
continuation from spacelike to timelike $k^{2}$. However, this is not the case. The parameter in which we are making an asymptotic approximation is not $k^{2}$ but rather $\zeta\left(k^{2}\right)$ and to analyze Stokes phenomena we have to ask how the analytic continuation from spacelike to timelike $k^{2}$ (again for fixed, small $g$ ) is mapped to a path in the complex $\zeta$ plane. One can see that a path in the $k^{2}$ plane from the negative real axis to the positive real axis, holding $\left|k^{2}\right|>\left(2 M_{\mathrm{cl}}\right)^{2}$ fixed and varying its phase from $\pi$ to 0 , corresponds to a path in the $\zeta$ plane which starts and ends on the positive real axis without crossing the anti-Stokes ray of $\zeta$, at $\operatorname{Re} \zeta=0$.

- Interestingly, the precise expression for the argument $\zeta$ of the Fourier transform of the soliton profile, (4.19), has appeared in the literature in the context of relativistic form factors - for the sine-Gordon model in [28] and for the Skyrme model in [19, 29]. In these references, one boosts the nonrelativistic form factor to the Breit frame. The latter, defined by $\mathbf{P}_{i}=-\mathbf{P}_{f}$, is the unique frame for which the magnitude of the spatial momentum is the same as the magnitude of the Lorentz momentum, $|\mathbf{k}|=\sqrt{-k^{2}}$. Although this procedure seemingly produces Lorentz-invariant results, no justification is given for choosing the Breit frame, or any frame for that matter. In contrast, our method remains Lorentz covariant at all stages and accounts for all relativistic corrections at leading order in the semiclassical expansion.

\section{Instanton-solitons in 5D MSYM}

The derivation of the suppression factor eq. (4.20) has so far been for purely scalar theories. Extending it to include more general Lagrangians with gauge fields and fermions introduces technical complications related to gauge invariance (gauge zero modes, ghosts). However, the simplicity of the answer, as well as the generality of the arguments used and the agreement with expectations from dimensional analysis point to a universal behavior. As a result, it is compelling to apply this framework to the interesting case of instanton-solitons in maximally supersymmetric 5D Yang-Mills (MSYM) theory.

Yang-Mills theory in 5D is normally viewed as an effective field theory, valid at low energies. However, the connection of 5D MSYM to the $(2,0)$ SCFT in 6D leaves open the possibility that this theory is in fact well defined [6, 7], even though it is perturbatively divergent at six loops $[8] .{ }^{10}$ In line with the rest of this letter, we argue that before sending the cutoff to infinity and declaring 5D MSYM to be UV-divergent, one has to also investigate contributions associated with soliton-antisoliton pair production. We stress that in doing so we are not treating this theory as an effective theory in the Wilsonian sense.

Instanton-solitons in 5D MSYM are finite-energy $\frac{1}{2}$-BPS field configurations. They solve the selfduality equation for the gauge field strength in the four spatial directions and as such are described by conventional 4D instanton solutions. For topological charge $c_{2}(F)=1$ and $\mathrm{SU}(2)$ gauge group the classical gauge field is given by

$$
A_{i}=U(\vec{\theta})^{-1}\left(\frac{\eta_{i j}^{a}(\mathbf{x}-\mathbf{X})^{j}}{(\mathbf{x}-\mathbf{X})^{2}+\rho^{2}} T^{a}\right) U(\vec{\theta}), \quad A_{0}=0,
$$

\footnotetext{
${ }^{10}$ Some nontrivial results compatible with this conjecture include [30-43].
} 
with $a=1,2,3, i=1, \ldots, 4$ and $\eta_{i j}^{a}$ the 't Hooft symbols. This solution has eight moduli: four center-of-mass collective coordinates $\mathbf{X}$, a size modulus $\rho$ and three Euler angles $\vec{\theta}$ parameterizing global gauge transformations. The associated moduli space is a hyperkähler manifold

$$
\mathcal{M}=\mathbb{R}^{4} \times \mathbb{R}_{+} \times S^{3} / \mathbb{Z}_{2}
$$

with metric

$$
\mathrm{d} s^{2}=\frac{4 \pi^{2}}{g_{\mathrm{YM}}^{2}}\left[\delta_{i j} \mathrm{~d} \mathbf{X}^{i} \mathrm{~d} \mathbf{X}^{j}+2\left(\mathrm{~d} \rho^{2}+\rho^{2} \tilde{G}_{\alpha \beta} \mathrm{d} \theta^{\alpha} \mathrm{d} \theta^{\beta}\right)\right],
$$

where $\tilde{G}_{\alpha \beta}$ is the metric on $\mathrm{SO}(3) \cong S^{3} / \mathbb{Z}_{2}$, the group of effective global gauge transformations, and $g_{\mathrm{YM}}^{2}$ has dimensions of length.

The presence of a noncompact size modulus translates into instanton-solitons that can have arbitrarily small or large sizes. Naive application of (4.20) would then imply that instanton-soliton pair production need not be suppressed relative to perturbative processes. However, this non-compact direction in the centered moduli space $\tilde{\mathcal{M}}$ also results in the corresponding Hamiltonian not admitting $L^{2}$-normalizable eigenfunctions. The centered Hamiltonian does possess a continuum of plane-wave normalizable wavefunctions, but this renders the interpretation of instanton-solitons as asymptotic states confusing, since they would correspond to particles with a continuously infinite number of internal degrees of freedom.

Moreover, the parameter controlling the semiclassical expansion of the Hamiltonian in the soliton sector is in fact $g^{2}=g_{\mathrm{YM}}^{2} / \rho$, which coincides with $R_{C} / R_{S}$. In particular, note that $g=g(\rho)$ is now moduli dependent. In the context of the semiclassical expansion (3.1), or more appropriately the relativistic version in appendix B, we can imagine a fixed $\rho$ such that $g(\rho)$ is small. However, when evaluating amplitudes, where one must integrate over all sizes, the semiclassical approximation breaks down. Consequently, the small-sized instanton-solitons invalidate our argument for exponential suppression.

One can attempt to circumvent this conclusion by turning on a scalar VEV, $\langle\Phi\rangle \neq 0$, and going out onto the Coulomb branch. ${ }^{11}$ It is known that in this case finding instantonsoliton solutions requires turning on an electric field, which stabilizes the classical size [4446]. From the point of view of the quantum theory, turning on an electric field generates a potential on the centered moduli space,

$$
\delta \mathrm{v}\left(U^{m}\right)=\frac{2 \pi^{2}}{g_{\mathrm{YM}}^{2}}\langle\Phi\rangle^{2} \rho^{2},
$$

and lifts the flat direction associated with the instanton-soliton size. Although $\rho$ is no longer a true modulus, the VEV provides an additional dimensionless parameter, $\epsilon:=g_{\mathrm{YM}}^{2}\langle\Phi\rangle$, that can be adjusted so that we remain in the small-potential approximation (4.1), where it is still appropriate to represent states as $L^{2}$-wavefunctions on $\tilde{\mathcal{M}}$. In order to determine the

\footnotetext{
${ }^{11}$ Here $\Phi$ is one of the five adjoint scalars of 5D SYM and should not be confused with the scalar fields for the linear sigma models considered in the previous sections.
} 
precise form of the resulting $L^{2}$-wavefunctions, one would need to compute the centeredmoduli-space Hamiltonian $\tilde{H}_{\text {eff }}$, appearing in (4.7) and (4.8). ${ }^{12}$

Our formalism has been general enough to accommodate such potentials on moduli space. Thus, despite the classical stabilization, one must still integrate over all of moduli space, which includes arbitrarily small sizes. However, as we have already discussed, this means treating the solitons semiclassically when $\rho \sim O\left(g_{\mathrm{YM}}^{2}\right)$, which is not valid because quantum corrections that have been neglected become important. Hence, turning on the potential (5.4) does not enable one to salvage an argument for faster-than-any-power suppression.

While none of these arguments definitively show that instanton-soliton contributions are not suppressed compared to perturbative processes, they at least allow for that possibility. Non-suppression of the pair-production amplitude would provide a mechanism via which the contribution of virtual soliton-antisoliton pairs to perturbative processes such as (2.2) can compete with the contribution from loops of perturbative particles. Such a mechanism is precisely what is called for in order to avoid contradicting the assumption of finiteness: one would require that the soliton-antisoliton contribution be divergent, with exactly the right coefficient to cancel the divergence found in [8]. This is an intriguing possibility, the investigation of which would, however, require an alternative approach to the one used here.

\section{Acknowledgments}

We would like to thank Philip Argyres, Tom Banks, Shabnam Beheshti, Jacques Distler, Michael Douglas, Daniel Friedan, Jeff Harvey, Daniel Jafferis, Seok Kim, Ilarion Melnikov, Greg Moore, John Schwarz and Edward Witten helpful discussions and comments. CP is a Royal Society Research Fellow and partly supported by the U.S. Department of Energy under grants DOE-SC0010008, DOE-ARRA-SC0003883 and DOE-DE-SC0007897. ABR is supported by the Mitchell Family Foundation. We would like to thank the Mitchell Institute at Texas A\&M and the NHETC at Rutgers University respectively for hospitality during the course of this work. We would also like to acknowledge the Aspen Center for Physics and NSF grant 1066293 for a stimulating research environment.

\section{A One-point function and the source-free e.o.m.}

Suppose we add sources $(J, K)$ for $(\chi, \pi)$ to the Lagrangian $(4.2)$ :

$$
L \rightarrow L+\epsilon \int \mathrm{d} \mathbf{x}(J \chi+K \pi)
$$

Here we have introduced a small parameter $\epsilon$; we will solve the classical equations of motion with source perturbatively in $\epsilon$. It should be sufficient to treat the sources in this fashion

\footnotetext{
${ }^{12}$ One will actually have a supersymmetric quantum mechanics with 8 supercharges so the wavefunctions will be forms or bispinors on the moduli space, due to realizing the fermi collective coordinate anticommutator as a Clifford algebra [47].
} 
since we are only interested in $n$-point correlators for which one only needs the behavior of the partition function in a neighborhood of $J=0=K$. We make a series expansion

$$
\chi_{\mathrm{cl}}=\chi_{\mathrm{cl}}^{(0)}+\epsilon \chi_{\mathrm{cl}}^{(1)}+O\left(\epsilon^{2}\right), \quad \pi_{\mathrm{cl}}=\pi_{\mathrm{cl}}^{(0)}+\epsilon \pi_{\mathrm{cl}}^{(1)}+O\left(\epsilon^{2}\right),
$$

and plug into the classical equations of motion with source. We then have to expand in fluctuations around the classical solution, $\chi=\chi_{\mathrm{cl}}+\delta \chi$. The contributions from the fluctuations are suppressed in powers of $g$.

Demanding that the classical equations hold order by order in $\epsilon$, we find that $\left(\chi_{\mathrm{cl}}^{(0)}, \pi_{\mathrm{cl}}^{(0)}\right)$ should solve the source-free equations of motion. The $\left(\chi_{\mathrm{cl}}^{(1)}, \pi_{\mathrm{cl}}^{(1)}\right)$ solve an inhomogeneous linear differential equation, involving the operator that controls the spectrum around the soliton. Let us denote the restriction of that operator to the space orthogonal to the zero modes by $\mathcal{O}$ (so that $\mathcal{O}$ has an inverse). We assume that $(J, K)$ have no overlap with the zero modes. Then the solution to $O\left(\epsilon^{2}\right)$ is

$$
\left(\begin{array}{c}
\chi_{\mathrm{cl}} \\
\pi_{\mathrm{cl}}
\end{array}\right)=\left(\begin{array}{c}
\chi_{\mathrm{cl}}^{(0)} \\
\pi_{\mathrm{cl}}^{(0)}
\end{array}\right)+\epsilon \mathcal{O}^{-1}\left(\begin{array}{c}
J \\
K
\end{array}\right)+O\left(\epsilon^{2}\right) .
$$

Now we must plug this back into the Lagrangian with source. Notice that, crucially, because $\left(\chi_{\mathrm{cl}}^{(0)}, \pi_{\mathrm{cl}}^{(0)}\right)$ satisfy the source-free equations of motion, the original Lagrangian has an expansion $L\left[\chi_{\mathrm{cl}}, \pi_{\mathrm{cl}}\right]=L_{\mathrm{cl}}^{(0)}+O\left(\epsilon^{2}\right)$; there are no linear terms in the source. Similarly, since we are expanding around a solution $\left(\chi_{\mathrm{cl}}, \pi_{\mathrm{cl}}\right)$ to the equations of motion, the first corrections from the quantum fluctuations come at quadratic order in $(\delta \chi, \delta \pi)$. Hence, the only linear terms in the source come from $\left(\chi_{\mathrm{cl}}^{(0)}+\delta \chi, \pi_{\mathrm{cl}}^{(0)}+\delta \pi\right)$ multiplying $(J, K)$ in the source term itself:

$$
\begin{aligned}
L+\int \mathrm{d} \mathbf{x}(J \chi+K \pi)= & L_{\mathrm{cl}}^{(0)}+\int \mathrm{d} \mathbf{x}\left(J\left(\chi_{\mathrm{cl}}^{(0)}+\delta \chi\right)+K\left(\pi_{\mathrm{cl}}^{(0)}+\delta \pi\right)\right) \\
& +O\left(J^{2}, K^{2}, \delta \chi^{2}, \delta \pi^{2}\right),
\end{aligned}
$$

where we have reabsorbed $\epsilon$ into the sources.

Hence, the one-point function for e.g. the $\chi$-field is

$$
\begin{aligned}
& \left.\frac{\delta}{\delta J}\left(\int[D \chi D \pi] \delta\left(F_{1}\right) \delta\left(F_{2}\right) e^{i \int \mathrm{d} t^{\prime}(L+J \chi+K \pi)}\right)\right|_{J=0=K} \\
& \quad=\int[D \chi D \pi] \delta\left(F_{1}\right) \delta\left(F_{2}\right)\left(\chi_{\mathrm{cl}}^{(0)}+\delta \chi\right) e^{i \int \mathrm{d} t^{\prime}\left(L_{\mathrm{cl}}^{(0)}+O\left(\delta \chi^{2}, \delta \pi^{2}\right)\right)} \\
& \quad=e^{i \int \mathrm{d} t^{\prime} L_{\mathrm{cl}}^{(0)}} \chi_{\mathrm{cl}}^{(0)} \int[D \delta \chi D \delta \pi] \delta\left(F_{1}[\delta \chi]\right) \delta\left(F_{2}[\delta \pi]\right) e^{O\left(\delta \chi^{2}, \delta \pi^{2}\right)}\left(1+\delta \chi / \chi_{\mathrm{cl}}^{(0)}\right)
\end{aligned}
$$

where the ratio $\delta \chi / \chi_{\mathrm{cl}}^{(0)}$ is $O(g)$. The Gaussian integral over the fluctuations gives a oneloop correction to $L_{\mathrm{cl}}^{(0)}$, which also only depends on $\chi_{\mathrm{cl}}^{(0)}, \pi_{\mathrm{cl}}^{(0)}$. As a result, the leading saddle-point contribution to the one-point function is expressed entirely in terms of the classical solution to the source-free equations of motion. 


\section{B The $\chi, \pi$ saddle point in the presence of centered moduli}

In this appendix we would like to find the saddle-point solutions to the $\chi, \pi$ equations of motion coming from the Hamiltonian ${ }^{13}$

$$
H=\frac{g^{4}}{2} a^{M} G_{M N} a^{N}+\mathrm{v}(U)+\int\left[\frac{1}{2} \pi \cdot \pi+g s \cdot \chi+\frac{1}{2} \chi \cdot \Delta \chi+V_{I}(\chi)\right]+O\left(g^{2}\right),
$$

with

$$
\begin{aligned}
& s\left(\mathbf{x} ; U^{m}\right):=\frac{1}{g^{2}}\left(-\partial_{\mathbf{x}}^{2} \phi+\left.\frac{\partial V}{\partial \Phi}\right|_{\Phi=\phi}\right), \quad \Delta:=-\delta_{a b} \partial_{\mathbf{x}}^{2}+\left.\frac{\delta^{2} V}{\delta \Phi \delta \Phi}\right|_{\Phi=\phi}, \\
& \mathrm{v}\left(U^{m}\right):=\frac{1}{g^{2}} \int \mathrm{d} \mathbf{x}\left(\frac{1}{2} \partial_{\mathbf{x}} \phi \cdot \partial_{\mathbf{x}} \phi+V(\phi)\right)=M_{\mathrm{cl}}+\delta \mathrm{v}\left(U^{m}\right) .
\end{aligned}
$$

The equations of motion are given by

$$
\dot{\chi}=\frac{\delta H}{\delta \pi}-\nu^{M} \partial_{M} \phi, \quad \dot{\pi}=-\frac{\delta H}{\delta \chi}-\mu^{M} \partial_{M} \phi,
$$

where the $\mu, \nu$ are Lagrange multupliers for the second-class constraints

$$
F_{1, M}:=\int \chi \cdot \partial_{M} \phi=0, \quad F_{2, M}:=\int \pi \cdot \partial_{M} \phi=0 .
$$

The equations we want to solve are

$$
\begin{aligned}
0= & \dot{\chi}+\nu^{M} \partial_{M} \phi-\pi+\partial_{M} \chi(C G C)^{M N}\left(P_{N}-\int \pi \cdot \partial_{N} \chi\right), \\
0= & \dot{\pi}+\mu^{M} \partial_{M} \phi+\frac{1}{g} \partial_{\mathbf{x}}^{2}(\phi+g \chi)-\frac{1}{g} V^{\prime}(\phi+g \chi) \\
& +g s-\partial_{M} \pi(C G C)^{M N}\left(P_{N}-\int \pi \cdot \partial_{N} \chi\right) \\
& \quad-g^{-1}\left(\partial_{M} \partial_{N} \phi\right) C^{M P}\left(P_{P}-\int \pi \cdot \partial_{P} \chi\right)(C G C)^{N Q}\left(P_{Q}-\int \pi \cdot \partial_{Q} \chi\right),
\end{aligned}
$$

where $C^{M N}=\left[(G-g \Xi)^{-1}\right]^{M N}$ and

$$
G_{M N}:=\frac{1}{g^{2}} \int \partial_{M} \phi \cdot \partial_{N} \phi, \quad \Xi_{M N}:=\frac{1}{g^{2}} \int \chi \cdot \partial_{M} \partial_{N} \phi .
$$

Our strategy will be to work in the following approximation scheme. On the one hand, after changing variables to the soliton-fixed frame, $\rho=\mathbf{x}-\mathbf{X}$, one finds that the equations do not depend on $\mathbf{X}$ and thus it is consistent to treat $\mathbf{P}$ as constant. On the other, the equations do depend on the relative moduli $U^{m}$ (through the dependence of the metric and the fields on them) so it is inconsistent to treat $P_{m}$ as constant. However, we will assume that the motion of the relative moduli is slowly varying, $\dot{U}^{m} \sim O(g)$, so that altogether

$$
\begin{aligned}
& \mathbf{P} \sim O\left(g^{-2}\right), \quad \dot{\mathbf{P}}=0, \quad P^{m} \sim O\left(g^{-1}\right) \\
& s\left(\mathbf{x} ; U^{m}\right) \sim O(1) \quad \Rightarrow \quad M_{\mathrm{cl}} \sim O\left(1 / g^{2}\right) \quad \text { and } \delta \mathrm{v}\left(U^{m}\right) \sim O(1) .
\end{aligned}
$$

\footnotetext{
${ }^{13}$ The saddle-point solutions that we will find here are the classical piece of the $\chi, \pi$ fields, previously denoted by $\chi_{\mathrm{cl}}, \pi_{\mathrm{cl}}$. We will drop the subscripts in the following equations for brevity.
} 
For example, note that in this approximation $\dot{\chi}=\partial_{m} \chi \dot{U}^{m}$ is suppressed by a factor of $g$ relative to $\chi$, since $\dot{U}^{m} \sim O(g)$.

We first solve for $\nu^{M}$ :

$$
\nu^{M}=G^{M N}(\Xi)_{N P}(C G C)^{P Q}\left(P_{Q}-\int \pi \cdot \partial_{Q} \chi\right)-G^{M N} \frac{1}{g^{2}} \int \partial_{N} \phi \cdot \dot{\chi}
$$

and then use that to find $\pi$ :

$$
\begin{aligned}
\pi=\Pi^{\perp} \dot{\chi}+\partial_{M} \phi G^{M N}(\Xi)_{N P}( & C G C)^{P Q}\left(P_{Q}-\int \pi \cdot \partial_{Q} \chi\right) \\
& +\partial_{M} \chi(C G C)^{M N}\left(P_{N}-\int \pi \cdot \partial_{N} \chi\right),
\end{aligned}
$$

where

$$
\Pi^{\perp} \dot{\chi}:=\dot{\chi}-\partial_{M} \phi G^{M N} \frac{1}{g^{2}} \int \partial_{N} \phi \cdot \dot{\chi}
$$

is the projection of $\dot{\chi}$ to the space orthogonal to the zero modes. Expressing $G^{-1} \Xi=$ $g^{-1}\left(\mathbb{1}-G^{-1} C\right)$, we can rewrite the above equation as

$$
\begin{aligned}
\pi=\Pi^{\perp} \dot{\chi}+\partial_{M} & \left(g^{-1} \phi+\chi\right)(C G C)^{M N}\left(P_{N}-\int \pi \cdot \partial_{N} \chi\right) \\
& -g^{-1} \partial_{M} \phi\left(G^{-1} C^{-1}\right)^{M}{ }_{P}(C G C)^{P N}\left(P_{N}-\int \pi \cdot \partial_{N} \chi\right)
\end{aligned}
$$

We will then assume our solution for $\chi$ is of the form

$$
\chi=\tilde{\chi}^{(-1)}-g^{-1} \phi+\tilde{\chi}^{(0)}
$$

where $\tilde{\chi}^{(-1)}$ and $\tilde{\chi}^{(0)}$ are $O\left(g^{-1}\right)$ and $O(1)$ terms respectively. Then the solution for $\pi$ can be expressed to leading order as

$$
\begin{aligned}
\pi=\Pi^{\perp} \dot{\tilde{\chi}}^{(-1)}+\left(\partial_{M} \tilde{\chi}^{(-1)}+\partial_{M} \tilde{\chi}^{(0)}\right. & \left.-g^{-1} \partial_{P} \phi\left(G^{-1} C^{-1}\right)^{P}{ }_{M}\right) D^{M N} \times \\
& \times\left(P_{N}-\int \Pi^{\perp} \dot{\tilde{\chi}}^{(-1)} \cdot \partial_{N} \tilde{\chi}^{(-1)}\right)+O(g) .
\end{aligned}
$$

Here we have defined a 'relativistic' moduli space metric

$$
D_{M N}=\int \partial_{M}\left(\tilde{\chi}^{(-1)}+\tilde{\chi}^{(0)}\right) \cdot \partial_{N}\left(\tilde{\chi}^{(-1)}+\tilde{\chi}^{(0)}\right)
$$

and in (B.12) it is understood that we only keep $D^{M N}, C^{M N}$ to the appropriate order in $g$, denoted e.g. by $D^{(n) M N}$.

Now let us turn to the $\chi$ equation, wich after various manipulations can be written as

$$
\begin{aligned}
E_{\chi}:= & {\left[\partial_{t}\left(\Pi^{\perp} \partial_{i} \tilde{\chi}^{(-1)}\right)+\partial_{i}\left(\Pi^{\perp} \dot{\tilde{\chi}}^{(-1)}\right)\right] D^{(2) i j} P_{j} } \\
& +\partial_{M} \partial_{N}\left(\tilde{\chi}^{(-1)}+\tilde{\chi}^{(0)}\right) D^{M P} D^{N Q} \times \\
& \quad \times\left(P_{P}-\int \Pi^{\perp} \dot{\tilde{\chi}}^{(-1)} \cdot \partial_{P} \tilde{\chi}^{(-1)}\right)\left(P_{Q}-\int \Pi^{\perp} \dot{\tilde{\chi}}^{(-1)} \cdot \partial_{Q} \tilde{\chi}^{(-1)}\right) \\
& -\partial_{\mathbf{x}}^{2}\left(\tilde{\chi}^{(-1)}+\tilde{\chi}^{(0)}\right)+g^{-1} V^{\prime}\left(g\left(\tilde{\chi}^{(-1)}+\tilde{\chi}^{(0)}\right)\right)+g s-\mu^{M} \partial_{M} \phi+O(g) .
\end{aligned}
$$


We can organize and solve this order by order in the coupling. At leading order we recover the expression

$$
E_{\chi}^{(-1)}=\left(\partial_{i} \partial_{j} \tilde{\chi}^{(-1)}\right) D^{(2) i k} D^{(2) j l} P_{k} P_{l}-\partial_{\mathbf{x}}^{2} \tilde{\chi}^{(-1)}+g^{-1} V\left(g \tilde{\chi}^{(-1)}\right)-\mu^{(-1) M} \partial_{M} \phi .
$$

Setting $\mu^{(-1) M}=0$ leads to the $D$-dimensional generalization of the similar equation in [20], the solution to which is given by the boosted soliton profile

$$
\tilde{\chi}^{(-1)}=g^{-1} \phi\left(\Lambda_{j}^{i}\left(x^{j}-X^{j}\right) ; U^{m}\right),
$$

where $\phi\left(\mathbf{x}-\mathbf{X} ; U^{m}\right)$ is the static soliton solution and

$$
\Lambda^{i}{ }_{j}=\delta^{i}{ }_{j}+\left(\sqrt{1+\frac{\mathbf{P}^{2}}{M_{\mathrm{cl}}^{2}}}-1\right) \frac{P^{i} P_{j}}{\mathbf{P}^{2}} .
$$

Regarding the equation of motion for the leading-order Lagrange multiplier $\mu^{(-1) M}$, we assume that $\chi,(B .11)$ with (B.16), satisfies the orthogonality constraint $\int \chi \cdot \partial_{M} \phi=0$ to leading order. The case of the kink in $\Phi^{4}$ theory is consistent with this condition. If the orthogonality condition on $\chi$ does not hold, then one should reinstate a nonzero $\mu^{(-1) M}$ and solve the coupled equations.

By further manipulating the $O(1)$ terms in (B.14) we can write the equation at this order in terms of a (linearized) differential operator

$$
\begin{aligned}
\mathcal{L}\left[\tilde{\chi}^{(0)}, \mu^{(0) M}\right]:= & -\left(\partial_{i} \partial_{j} \tilde{\chi}^{(0)}\right) D^{(2) i k} D^{(2) j l} P_{k} P_{l}+2\left(\partial_{i} \partial_{M} \tilde{\chi}^{(-1)}\right) D^{(2) i j} D^{(3) M k} P_{j} P_{k} \\
& -\partial_{\mathbf{x}}^{2} \tilde{\chi}^{(0)}+V^{\prime \prime}\left(g \tilde{\chi}^{(-1)}\right) \tilde{\chi}^{(0)}-\mu^{(0) M} \partial_{M} \phi
\end{aligned}
$$

and the source term

$$
\begin{aligned}
\mathcal{S}\left[\tilde{\chi}^{(-1)}\right]:= & -\left[\partial_{t}\left(\Pi^{\perp} \partial_{i} \tilde{\chi}^{(-1)}\right)+\partial_{i}\left(\Pi^{\perp} \dot{\tilde{\chi}}^{(-1)}\right)\right] D^{(2) i j} P_{j}-g s \\
& +2\left(\partial_{i} \partial_{j} \tilde{\chi}^{(-1)}\right) D^{(2) i k} D^{(2) j l} P_{k} \int \Pi^{\perp} \dot{\tilde{\chi}}^{(-1)} \cdot \partial_{l} \tilde{\chi}^{(-1)} \\
& -2\left(\partial_{i} \partial_{m} \tilde{\chi}^{(-1)}\right) D^{(2) i j} D^{(2) m n} P_{j}\left(P_{n}-\int \Pi^{\perp} \dot{\tilde{\chi}}^{(-1)} \cdot \partial_{n} \tilde{\chi}^{(-1)}\right),
\end{aligned}
$$

such that

$$
E_{\chi}^{(0)}=\mathcal{L}\left[\tilde{\chi}^{(0)}, \mu^{(0) M}\right]-\mathcal{S}\left[\tilde{\chi}^{(-1)}\right]
$$

This can be formally solved by taking

$$
\left(\tilde{\chi}^{(0)}, \mu^{(0) M}\right)=\mathcal{L}^{-1} \mathcal{S}\left[\tilde{\chi}^{(-1)}\right] .
$$

Open Access. This article is distributed under the terms of the Creative Commons Attribution License (CC-BY 4.0), which permits any use, distribution and reproduction in any medium, provided the original author(s) and source are credited. 


\section{References}

[1] J. Le Guillou and J. Zinn-Justin, Large order behaviour of perturbation theory, Current Physics Sources and Comments volume 7, North-Holland, The Netherlands (1990).

[2] A.K. Drukier and S. Nussinov, Monopole pair creation in energetic collisions: is it possible?, Phys. Rev. Lett. 49 (1982) 102 [INSPIRE].

[3] C. Bachas, On the breakdown of perturbation theory, Theor. Math. Phys. 95 (1993) 491 [hep-th/9212033] [INSPIRE].

[4] T. Banks, Arguments against a finite $N=8$ supergravity, arXiv:1205.5768 [INSPIRE].

[5] S.R. Coleman, Crossing symmetry in semiclassical soliton scattering, Phys. Rev. D 12 (1975) 1650 [INSPIRE].

[6] M.R. Douglas, On D = 5 super Yang-Mills theory and (2,0) theory, JHEP 02 (2011) 011 [arXiv:1012.2880] [INSPIRE].

[7] N. Lambert, C. Papageorgakis and M. Schmidt-Sommerfeld, M5-branes, D4-branes and Quantum 5D super-Yang-Mills, JHEP 01 (2011) 083 [arXiv:1012.2882] [INSPIRE].

[8] Z. Bern et al., D = 5 maximally supersymmetric Yang-Mills theory diverges at six loops, Phys. Rev. D 87 (2013) 025018 [arXiv: 1210.7709] [INSPIRE].

[9] J. Goldstone and R. Jackiw, Quantization of nonlinear waves, Phys. Rev. D 11 (1975) 1486 [INSPIRE].

[10] G.H. Derrick, Comments on nonlinear wave equations as models for elementary particles, J. Math. Phys. 5 (1964) 1252 [InSPIRE].

[11] N. Manton and P. Sutcliffe, Topological solitons, Cambridge Monographs on Mathematical Physics. Cambridge University Press, Cambridge U.K. (2004).

[12] R. Rajaraman and E.J. Weinberg, Internal symmetry and the semiclassical method in quantum field theory, Phys. Rev. D 11 (1975) 2950 [INSPIRE].

[13] D. Bazeia, J.R.S. Nascimento, R.F. Ribeiro and D. Toledo, Soliton stability in systems of two real scalar fields, J. Phys. A 30 (1997) 8157 [hep-th/9705224] [INSPIRE].

[14] G.P. de Brito and A. de Souza Dutra, Orbit based procedure for doublets of scalar fields and the emergence of triple kinks and other defects, Phys. Lett. B 736 (2014) 438 [arXiv: 1405.5458] [INSPIRE].

[15] S.V. Demidov and D.G. Levkov, Soliton-antisoliton pair production in particle collisions, Phys. Rev. Lett. 107 (2011) 071601 [arXiv:1103.0013] [INSPIRE].

[16] S.V. Demidov and D.G. Levkov, Soliton pair creation in classical wave scattering, JHEP 06 (2011) 016 [arXiv:1103.2133] [INSPIRE].

[17] R.J. Eden, P.V. Landshoff, D.I. Olive and J.C. Polkinghorne, The analytic S-matrix, Cambridge University Press, Cambridge U.K. (1966).

[18] C. Papageorgakis and A.B. Royston, Scalar soliton quantization with generic moduli, JHEP 06 (2014) 003 [arXiv: 1403.5017] [INSPIRE].

[19] X.-D. Ji, A relativistic skyrmion and its form-factors, Phys. Lett. B 254 (1991) 456 [INSPIRE].

[20] J.-L. Gervais, A. Jevicki and B. Sakita, Perturbation expansion around extended particle states in quantum field theory. 1, Phys. Rev. D 12 (1975) 1038 [INSPIRE]. 
[21] N. Dorey, M.P. Mattis and J. Hughes, Soliton quantization and internal symmetry, Phys. Rev. D 49 (1994) 3598 [hep-th/9309018] [INSPIRE].

[22] N. Dorey, J. Hughes and M.P. Mattis, Skyrmion quantization and the decay of the Delta, Phys. Rev. D 50 (1994) 5816 [hep-ph/9404274] [INSPIRE].

[23] C. Teitelboim, Proper time approach to the quantization of the gravitational field, Phys. Lett. B 96 (1980) 77 [INSPIRE].

[24] M. Henneaux and C. Teitelboim, Relativistic quantum mechanics of supersymmetric particles, Annals Phys. 143 (1982) 127 [InSPIRE].

[25] P.D. Mannheim, Klein-Gordon propagator via first quantization, Phys. Lett. B 166 (1986) 191 [INSPIRE].

[26] S. Monaghan, BRS hamiltonian quantization of a spinless relativistic particle in relativistic gauges, Phys. Lett. B 178 (1986) 231 [INSPIRE].

[27] C. Papageorgakis and A.B. Royston, A semiclassical relativistic form factor for the kink, in preparation.

[28] P.H. Weisz, Exact quantum sine-Gordon soliton form-factors, Phys. Lett. B 67 (1977) 179 [INSPIRE].

[29] G. Holzwarth, Electromagnetic nucleon form-factors and their spectral functions in soliton models, Z. Phys. A 356 (1996) 339 [hep-ph/9606336] [INSPIRE].

[30] Y. Tachikawa, On S-duality of 5D super Yang-Mills on $S^{1}$, JHEP 11 (2011) 123 [arXiv:1110.0531] [INSPIRE].

[31] N. Lambert, H. Nastase and C. Papageorgakis, 5D Yang-Mills instantons from ABJM monopoles, Phys. Rev. D 85 (2012) 066002 [arXiv:1111.5619] [InSPIRE].

[32] H.-C. Kim, S. Kim, E. Koh, K. Lee and S. Lee, On instantons as Kaluza-Klein modes of M5-branes, JHEP 12 (2011) 031 [arXiv:1110.2175] [INSPIRE].

[33] D. Young, Wilson loops in five-dimensional super-Yang-Mills, JHEP 02 (2012) 052 [arXiv: 1112.3309] [INSPIRE].

[34] J. Källén and M. Zabzine, Twisted supersymmetric 5D Yang-Mills theory and contact geometry, JHEP 05 (2012) 125 [arXiv:1202.1956] [INSPIRE].

[35] K. Hosomichi, R.-K. Seong and S. Terashima, Supersymmetric gauge theories on the five-sphere, Nucl. Phys. B 865 (2012) 376 [arXiv:1203.0371] [InSPIRE].

[36] J. Källén, J. Qiu and M. Zabzine, The perturbative partition function of supersymmetric $5 D$ Yang-Mills theory with matter on the five-sphere, JHEP 08 (2012) 157 [arXiv:1206.6008] [INSPIRE].

[37] H.-C. Kim and S. Kim, M5-branes from gauge theories on the 5-sphere, JHEP 05 (2013) 144 [arXiv:1206.6339] [INSPIRE].

[38] J. Källén, J.A. Minahan, A. Nedelin and M. Zabzine, $N^{3}$-behavior from 5D Yang-Mills theory, JHEP 10 (2012) 184 [arXiv:1207.3763] [INSPIRE].

[39] D. Bak and A. Gustavsson, M5/D4 brane partition function on a circle bundle, JHEP 12 (2012) 099 [arXiv:1209.4391] [INSPIRE].

[40] Y. Fukuda, T. Kawano and N. Matsumiya, 5D SYM and 2D q-deformed YM, Nucl. Phys. B 869 (2013) 493 [arXiv:1210.2855] [INSPIRE]. 
[41] H.-C. Kim, J. Kim and S. Kim, Instantons on the 5-sphere and M5-branes, arXiv: 1211.0144 [INSPIRE].

[42] N. Lambert, C. Papageorgakis and M. Schmidt-Sommerfeld, Deconstructing $(2,0)$ proposals, Phys. Rev. D 88 (2013) 026007 [arXiv:1212.3337] [InSPIRE].

[43] D. Bak and A. Gustavsson, One dyonic instanton in 5D maximal SYM theory, JHEP 07 (2013) 021 [arXiv: 1305.3637] [INSPIRE].

[44] N.D. Lambert and D. Tong, Dyonic instantons in five-dimensional gauge theories, Phys. Lett. B 462 (1999) 89 [hep-th/9907014] [INSPIRE].

[45] K. Peeters and M. Zamaklar, Motion on moduli spaces with potentials, JHEP 12 (2001) 032 [hep-th/0107164] [INSPIRE].

[46] J.P. Allen and D.J. Smith, The low energy dynamics of charge two dyonic instantons, JHEP 02 (2013) 113 [arXiv:1210.3208] [INSPIRE].

[47] D. Bak, C.-k. Lee, K.-M. Lee and P. Yi, Low-energy dynamics for 1/4 BPS dyons, Phys. Rev. D 61 (2000) 025001 [hep-th/9906119] [InSPIRE]. 